[CONTRIBUTION FROM THE ELECTROCHEMICAL LABORATORIES OF THE UNIVERSTTY OF MANCHESTER.]

\title{
REPLY TO HARDING AND SMITH'S COMMENTS ON NEW- BERY'S CRITICISM.
}

By EDGAR NEWBERY.

Received October 7, 1919.

The most important objection raised by Professor Smith and Dr. Harding to my suggestion that the overvoltage hydrides and higher oxides are responsible for the changes of electrical resistance observed by them, lies in the supposition that these compounds have a higher electrical conductivity than has the metal itself. Personally, I do not believe that such conductivity of compounds is possible and I am unable to agree with the above authors that the postulation of this high conductivity is at all necessary.

The first application of a potential difference between the wire and the surrounding medium produces an electrical double layer which must exert enormous mechanical pressure upon the outer layers of the wire. This pressure will not only close up some of the cavities already formed in a previously treated wire, but will even compress the crystals of an untreated wire into closer contact, thus increasing the conductivity. This lateral pressure on the wire would naturally tend to increase its length, which is in accordance with the observations of Harding and Smith.

In the case of palladium the rate of absorption of hydrogen is so rapid that this initial increase of conductivity is not observable with an untreated wire, although it is readily observed when oxygen is liberated. Hydride formation does not occur until after the surface is saturated with dissolved hydrogen.

This "electro-striction" effect is quite outside the hydride hypothesis and occurs before hydrides have time to form. This, perhaps, meets the objections raised by Professor Smith amd Dr. Harding in the first 4 paragraphs of the preceding paper. The fall of resistance referred to in the fifth paragraph is evidently due to the rapid reduction of oxide to metal.

My most serious objection to Smith and Harding's theory may be stated bluntly as follows: If $80 \mathrm{cc}$. of atomic hydrogen introduced into metallic palladium increases its resistance to a degree roughly proportional to the quantity of gas absorbed, why should a further $20 \mathrm{cc}$. of the same gas reduce the resistance? My belief is that the last $20 \mathrm{cc}$. does not exist in the form of gas, but is chemically combined with the palladium and this belief is based on the fact that its electrolytic potential is widely different (up to 0.7 volt) from that of the $80 \mathrm{cc}$. of monatomic hydrogen first introduced. Smith and Harding's theory entirely fails to account both for the constancy of the potential up to this point and for this sudden and very great change in electrolytic potential which occurs simul- 
taneously with the fall of resistance. The mere accumulation of an extra $20 \%$ of a gas already present is quite inadequate to explain this. Also, the fact that the voltage attained is determined by the valence of the metal can hardly be reconciled with any theory which does not involve chemical compounds.

To test my views of the possibility of electro-striction affecting the resistance of the wire I would suggest that Prof. Smith and Dr. Harding should make the following simple experiment which I am unfortunately unable to make for myself at the present moment: A long, fine wire of platinum or palladium should be coiled closely round a mica plate (a platinum resistance thermometer would do very well) and its resistance measured when immersed in various electrolytes-pure water, strong acid or alkali, and also in oil or benzene, as well as in air. ${ }^{1}$

One important point has been overlooked in the original experiments of Smith and Harding, namely the conductivity of the electrolyte. In their apparatus this is certainly appreciable and the highly complicating phenomena of transfer resistance are also present. A certain quantity of current must of necessity flow through the electrolyte alongside the wire. If the resistance of the circuit, wire-electrolyte-wire, were constant, the shape of the curves would be unaffected. Unfortunately, this is far from being the case. The transfer resistance opposing the passage of current between the wire and the electrolyte is a quantity which varies according to circumstances from o to over $1000 \mathrm{ohms}$ per sq. $\mathrm{cm}$., and the lower this transfer resistance, the greater the current carried by the electrolyte. It is quite impossible to predict what this transfer resistance will be, since it is dependent upon temperature, current density, time, material and condition of electrode, electrolyte, and specially upon the previous history of the electrode, and its variation is not regular with any of these quantities or qualities. The confusion introduced by this troublesome factor is great enough to invalidate both of the explanations suggested for Harding and Smith's observations, and its elimination promises to be equally troublesome. Experiments with very wide and very narrow tubes surrounding the wire and also with electrolytes of varying conductivity might indicate what allowances should be made. It is possible that with a very narrow surrounding tube and an electrolyte of high resistance, some features of the curve may tend to disappear. If so, these features may be safely put down to transfer resistance. Until some such experiments on these or similar lines have been carried out, it is perhaps unwise to attempt any further explanation of the observed phenomena. $^{2}$

1 This form of experiment is of course open to the same objections as those mentioned in the following section. Nevertheless it is worth trying.

2 An account of tranșfer resistance may be found in J. Faraday Soc., 15, I (1919), "The Resistance of an Electrolytic Cell." 
One further point may be mentioned with regard to the ninth paragraph of Harding and Smith's comment on my criticism. They refer to "a metallic compound existing within an alloy side by side with a solid solution from which it differs diametrically in properties, but from which it does not form a distinct phase."

I agree that such a hypothesis is very improbable and I had no idea of suggesting this. Though the application of the phase rule to such a system which is not in equilibrium is very unsafe, yet if we consider a very small volume in the interior of the metal where the changes are progressing slowly, the condition of equilibrium may approximately hold.

We have two components, metal and monatomic gas. Metal may be considered to have ceased to exist as pure metal and the phases present will be saturated solid solution, metallic hydride, free gas. If the pressure be increased, more gas will combine with the solid solution to form hydride. If it be decreased, hydride will decompose giving free gas and solid solution. The hydrides, therefore, form a distinct phase with a definite dissociation pressure for a given temperature, the whole process being exactly analogous to the decomposition of calcium carbonate by heat. The fact that hydrides are actually formed hardly admits of dispute, the evidence being almost overwhelming, ${ }^{1}$ while the formation of the corresponding oxides is a well established fact. The question at issue is not whether these compounds exist, but whether they are responsible for the resistance changes observed by Harding and Smith.

In conclusion, I desire to express my appreciation of the courtesy and broad-mindedness with which Professor Smith and Dr. Harding have met my criticisms and also my admiration of the delicacy and ingenuity of their apparatus and the work under discussion.

MANCHESTHR, ENGLAND.

[CONTRIBUTION hROM THE CheMtCAL LABORATORY OF PRINCETON UNIVERSTTY.]

COMMENT ON PROFESSOR NEWBERY'S REPLY.

By EARLE A. HaRding and Donald P. SMith.

Received October 7, 1919.

The first new explanation advanced in this reply assumes that the resistance of the palladium wire is reduced by an electrostrictive effect. Since, at $25^{\circ}$, the average pressure coefficient of the resistance of palladium ${ }^{2}$ is only $\longrightarrow .1887 \times 10^{-5}$, the electrostriction necessary to cause a change of resistance of $25 \%$ would be of a magnitude far beyond any which could be produced by the small voltages applied ( $<6$ volts). Moreover, this explanation assumes that the crosss section of the wire is reduced during

${ }^{1}$ See J. Chem. Soc., I09, I051, I066, I107, I359 (I916); IIr, 470 (I917); also Manchester Memoirs, 6r, No. 9 (1917).

- Bridgman, Proc. Am. Acad., 52, 612 (1916). 